\title{
On Exponential Interval Valued Intuitionistic Fuzzy Entropy of Order $\alpha$ and type $\beta$ and its Applications in Decision Making
}

\author{
Rajeev Kaushik, \\ Research Scholar, \\ Singhania University, \\ Pacheri Bari, Rajasthan, India.
}

\author{
Rakesh K Bajaj, \\ Associate Professor, Department of Mathematics, \\ JUIT, Waknaghat, \\ Solan, Himachal Pradesh, India.
}

\begin{abstract}
In the present paper, a new entropy of order $\alpha$ and type $\beta$ on Interval-Valued Intutionistic Fuzzy Sets (IVIFSs) along with their proofs of validity is proposed. It has been proved that the proposed entropy has monotonic decreasing behavior with respect to $\alpha$ and $\beta$. Further, a new algorithm for multiple attribute decision making method (MADM) has been provided using the benefit attributes and cost attribute weights on the proposed entropy, where the alternatives on attributes are expressed by interval-valued intuitionistic fuzzy sets (IVIFS). The information about attribute weight is unknown. Finally, numerical example for illustrating the proposed methodology has also been provided to illustrate the applicability and validity of the newly proposed method.
\end{abstract}

\section{Keywords:}

Interval valued intuitionistic fuzzy set (IVIFS), entropy, multi attribute decision making (MADM)

\section{INTRODUCTION}

Atanassov [1] generalized the Zadeh's fuzzy sets [3] and a higher order fuzzy set i.e. Intuitionistic fuzzy set (IFS) and later on Atanassov and Gorgav [2]further introduced the interval-valued Intuitionistic fuzzy sets (IVIFS). The characteristics of IVIFS are the values of its membership functions and non-membership functions which are intervals rather than exact numbers. Entropy of fuzzy set describes the fuzziness degree of a fuzzy set and was first mentioned by Zadeh [3] in 1965. In 1972 [6] Deluca and Termini presented some axioms to describe the fuzziness degree of fuzzy set, with which fuzzy entropy based on Shannon's function was proposed. Later on in 1975 Kaufmann [7] proposed a method for measuring the fuzziness degree of a fuzzy set by a metric distance between its membership function and the membership function of its nearest crisp set. As for Intuitionistic fuzzy set, Bustince and Burillo [4] firstly introduced an entropy on IFS in 1996, and then Hung [8], Zhang [9], Vlachos and Sergiadis [10], Zeng [11] presented different entropies on IFS from different aspects. Another method presented by Yager [12] was to view the fuzziness degree of a fuzzy set in terms of a lack of distinction between the fuzzy set and its complement. Atanassov introduced prominent form by combining the concept of IFS and IVFS i.e., IVIFS interval val- ued Intuitionistic fuzzy set [2] and this concept is widely used in multi criterion decision making problems [13], [14], [15], [16], [17]. Based on these concepts and their axiomatic definitions, Zeng and $\mathrm{Li}[18]$ investigated the relationship among inclusion measure, similarity measure, and fuzziness of fuzzy sets. A multiple attribute decision making (MADM) is used to find a most suitable solution from a finite number of feasible alternatives assessed on multiple attributes as defined by Liang, Zhang and Ding [19]. The decision maker must provide their preference for information in the form of numerical values, exact values, and interval-number values, FS, IFS and IVIFS to choose most desirable solution.

\section{INTERVAL-VALUED INTUTIONISTIC FUZZY SETS}

An interval-valued intuitionistic fuzzy $\operatorname{set}(\operatorname{IvIFS}) A$ in the finite universe $X$ is expressed by the form

$$
A=\left\{\left(x, \mu_{A}(x), \gamma_{A}(x)\right) \mid x \in X\right\},
$$

where $\mu_{A}: X \rightarrow \operatorname{Int}[0,1], \gamma_{A}: X \rightarrow \operatorname{Int}[0,1]$ along with the condition,

$$
0 \leq \sup \left(\mu_{A}(x)\right)+\sup \left(\gamma_{A}(x)\right) \leq 1, \forall x \in X .
$$

The interval $\mu_{A}(x)$ and $\gamma_{A}(x)$ specifies the membership degree and non-membership degree of $x$ to $A$ and $A$ is defined as:

$$
A=\left\{\left(x,\left[\mu_{A L}(x), \mu_{A U}(x)\right],\left[\gamma_{A L}(x), \gamma_{A U}(x)\right]\right) \mid x \in X\right\}
$$

Also for each element $x$, the unknown degree (hesitancy degree) of an IVIFS $A$ is defined as follows:

$$
\pi_{A}(x)=\left[1-\mu_{A U}(x)-\gamma_{A U}(x), 1-\mu_{A L}(x)-\gamma_{A L}(x)\right]
$$

\section{ENTROPY ON IVIFS}

In fuzzy set theory, the entropy is a measure of fuzziness which specifies the amount of average difficulty in making decision whether an element belongs to a set or not. Zadeh [3] in 1965 introduced the concept of entropy in fuzzy sets first and later in 1996 Bustince and Burillo [4] proposed the entropy in interval valued Intuitionistic fuzzy sets. Ying Jun Zhang, Pei Jun Ma, Xiao Hong Su, Chi Ping Zhang in 2011 [5] give the definitions of entropy on 
IVIFS based on the work of Zadeh \& Bustince and Burillo and then a different method to construct entropy on IVIFS is proposed.

Definition 1. Consider $A \in \operatorname{IVIFS}(X)$. A real-vlued function $H_{n}: \operatorname{IVIFS}(X) \rightarrow[0,1]$ is called the entropy on $A$ if $H(A)$ satisfies the following properties:

(1) $H(A)=0$ iff $A$ is a fuzzy set;

(2) $H(A)=1$ iff $\mu_{A}(X)=[0,0]$ and $\gamma_{A}(X)=[0,0]$, $\forall x \in X$

(3) $H(A)=H\left(A^{C}\right)$ for all $A \in \operatorname{IVIFS}(X)$;

(4) For two IVIFS $A$ and $B$ on $X$, if $A \subseteq B$ then $H(A) \geq H(B)$.

On the basis of the definition given above it can be concluded that IFS $A$ has maximum uncertainty when $\pi_{A}(X)=1$ for all $x \in X$ and has minimum uncertainty when $A$ reduces to a fuzzy set. As the definitions for the entropy in IFS and IVIFS are seemed to be similar, two parameters $\alpha$ and type $\beta$ are introduced and then an IVIFS entropy is constructed which is as under:-

$$
H_{\alpha}^{\beta}(A)=\frac{1}{n} \sum_{i=1}^{n}\left[1-\left(\tilde{\mu}_{A}\left(x_{i}\right)+\tilde{\gamma}_{A}\left(x_{i}\right)\right)^{\beta} e^{1-\left(\tilde{\mu}_{A}\left(x_{i}\right)+\tilde{\gamma}_{A}\left(x_{i}\right)\right)^{\beta}}\right],
$$

where $\tilde{\mu}_{A}\left(x_{i}\right)=\mu_{A L}\left(x_{i}\right)+\alpha \Delta \mu_{A}\left(x_{i}\right)$ and

$\tilde{\gamma}_{A}\left(x_{i}\right)=\gamma_{A L}\left(x_{i}\right)+\alpha \Delta \gamma_{A}\left(x_{i}\right)$,

Here $\Delta \mu_{A}\left(x_{i}\right)=\mu_{A U}\left(x_{i}\right)-\mu_{A L}\left(x_{i}\right)$,

$\Delta \gamma_{A}\left(x_{i}\right)=\gamma_{A U}\left(x_{i}\right)-\gamma_{A L}\left(x_{i}\right), \forall x \in X, \alpha \in[0,1]$.

Now, with the help of four axioms the above proposed entropy is proved.

(1) When $A$ is fuzzy set, then from (eq.5) the value of $H_{\alpha}^{\beta}(A)=$ 0 , which implies that, $\left(\tilde{\mu}_{A}\left(x_{i}\right)+\tilde{\gamma}_{A}\left(x_{i}\right)\right)^{\beta}=1$, where $\alpha \in$ $(0,1)$ and $\beta \in(0,1)$.

Substituting the values of $\tilde{\mu}_{A}$ and $\tilde{\gamma}_{A}$ from above, equation is equivalent to,

$$
\begin{gathered}
\left(\mu_{A L}\left(x_{i}\right)+\alpha \mu_{A U}\left(x_{i}\right)-\alpha \mu_{A L}\left(x_{i}\right)+\gamma_{A L}\left(x_{i}\right)\right. \\
\left.+\alpha \gamma_{A U}\left(x_{i}\right)-\alpha \gamma_{A L}\left(x_{i}\right)\right)^{\beta}=1 ; \\
\Rightarrow\left((1-\alpha) \mu_{A L}\left(x_{i}\right)+\alpha \mu_{A U}\left(x_{i}\right)\right. \\
\left.+(1-\alpha) \gamma_{A L}\left(x_{i}\right)+\alpha \gamma_{A U}\left(x_{i}\right)\right)^{\beta}=1 ; \\
\Rightarrow\left((1-\alpha)\left(\mu_{A L}\left(x_{i}\right)+\gamma_{A L}\left(x_{i}\right)\right)\right. \\
\left.+\alpha\left(\mu_{A U}\left(x_{i}\right)+\gamma_{A U}\left(x_{i}\right)\right)\right)^{\beta}=1 .
\end{gathered}
$$

Since

$$
0 \leq \mu_{A L}\left(x_{i}\right)+\gamma_{A L}\left(x_{i}\right) \leq \mu_{A U}\left(x_{i}\right)+\gamma_{A U}\left(x_{i}\right) \leq 1
$$

therefore,

$$
\begin{gathered}
(0)^{\beta} \leq\left(\mu_{A L}\left(x_{i}\right)+\gamma_{A L}\left(x_{i}\right)\right)^{\beta} \\
\leq\left(\mu_{A U}\left(x_{i}\right)+\gamma_{A U}\left(x_{i}\right)\right)^{\beta} \leq(1)^{\beta}, \\
\Rightarrow 0 \leq\left(\mu_{A L}\left(x_{i}\right)+\gamma_{A L}\left(x_{i}\right)\right)^{\beta} \leq\left(\mu_{A U}\left(x_{i}\right)+\gamma_{A U}\left(x_{i}\right)\right)^{\beta} \leq 1,
\end{gathered}
$$

and

$\left((1-\alpha)\left(\mu_{A L}\left(x_{i}\right)+\gamma_{A L}\left(x_{i}\right)\right)+\alpha\left(\mu_{A U}\left(x_{i}\right)+\gamma_{A U}\left(x_{i}\right)\right)\right)^{\beta}=1$.

Hold if,

$\left(\mu_{A L}\left(x_{i}\right)+\gamma_{A L}\left(x_{i}\right)\right)^{\beta}=1$ and $\left(\mu_{A U}\left(x_{i}\right)+\gamma_{A U}\left(x_{i}\right)\right)^{\beta}=1$.
If

$\left(\mu_{A L}\left(x_{i}\right)+\gamma_{A L}\left(x_{i}\right)\right)^{\beta} \prec 1$ and $\left(\mu_{A U}\left(x_{i}\right)+\gamma_{A U}\left(x_{i}\right)\right)^{\beta}=1$

Or

$\left(\mu_{A L}\left(x_{i}\right)+\gamma_{A L}\left(x_{i}\right)\right)^{\beta} \prec 1$ and $\left(\mu_{A U}\left(x_{i}\right)+\gamma_{A U}\left(x_{i}\right)\right)^{\beta} \prec 1$.

From this it is clear that $\left(\tilde{\mu}_{A}\left(x_{i}\right)+\tilde{\gamma}_{A}\left(x_{i}\right)\right)^{\beta} \prec 1$ which contradicts $\left(\tilde{\mu}_{A}\left(x_{i}\right)+\tilde{\gamma}_{A}\left(x_{i}\right)\right)^{\beta}=1$ therefore, as per the definition of IVIFS $\left(\mu_{A L}\left(x_{i}\right)+\gamma_{A L}\left(x_{i}\right)\right)^{\beta}=1$ shows given set $A$ is fuzzy set.

(2) According to equation given, we have $H_{\alpha}^{\beta}(A)=1$. which implies that,

$\left(\tilde{\mu}_{A}\left(x_{i}\right)+\tilde{\gamma}_{A}\left(x_{i}\right)\right)^{\beta}=0, \alpha \in(0,1)$ and $\beta \in(0,1) ;$

Since $0 \leq \tilde{\mu}_{A}\left(x_{i}\right)+\tilde{\gamma}_{A}\left(x_{i}\right) \leq 1$ therefore, it implies that $0 \leq\left(\tilde{\mu}_{A}\left(x_{i}\right)+\tilde{\gamma}_{A}\left(x_{i}\right)\right)^{\beta} \leq 1$.

Then, $\left(\tilde{\mu}_{A}\left(x_{i}\right)+\tilde{\gamma}_{A}\left(x_{i}\right)\right)^{\beta}=0$ is equivalent to

$$
\begin{aligned}
& \left((1-\alpha) \mu_{A L}\left(x_{i}\right)+\alpha \mu_{A U}\left(x_{i}\right)\right. \\
& \left.\quad+(1-\alpha) \gamma_{A L}\left(x_{i}\right)+\alpha \gamma_{A U}\left(x_{i}\right)\right)^{\beta}=0,
\end{aligned}
$$

and this equation holds if:

$\mu_{A L}\left(x_{i}\right)+\gamma_{A L}\left(x_{i}\right)=0$ and $\mu_{A U}\left(x_{i}\right)+\gamma_{A U}\left(x_{i}\right)=0$. and $\mu_{A L}\left(x_{i}\right)+\gamma_{A L}\left(x_{i}\right)=0$ and $\mu_{A L}\left(x_{i}\right)+\gamma_{A L}\left(x_{i}\right) \succ$ 0 and $\mu_{A U}\left(x_{i}\right)+\gamma_{A U}\left(x_{i}\right) \succ 0$ From this it is clear that, $\left(\tilde{\mu}_{A}\left(x_{i}\right)+\tilde{\gamma}_{A}\left(x_{i}\right)\right)^{\beta} \succ 0$,

which contradicts $\left(\tilde{\mu}_{A}\left(x_{i}\right)+\tilde{\gamma}_{A}\left(x_{i}\right)\right)^{\beta}=0$ and $\mu_{A U}\left(x_{i}\right)+$ $\gamma_{A U}\left(x_{i}\right)=0$,

$\Rightarrow \quad \mu_{A}\left(x_{i}\right)=[0,0]$ and $\gamma_{A}\left(x_{i}\right)=[0,0]$ for all $x \in X$.

(3) Let $A=\left\{\left(x,\left[\mu_{A L}\left(x_{i}\right), \mu_{A U}\left(x_{i}\right)\right],\left[\gamma_{A L}\left(x_{i}\right), \gamma_{A U}\left(x_{i}\right)\right]\right) \mid x \in X\right\}$, where

$$
\begin{gathered}
\mu_{A L}\left(x_{i}\right)=\inf \left(\mu_{A}\left(x_{i}\right)\right), \mu_{A U}\left(x_{i}\right)=\sup \left(\mu_{A}\left(x_{i}\right)\right), \\
\gamma_{A L}\left(x_{i}\right)=\inf \left(\gamma_{A}\left(x_{i}\right)\right) \text { and } \gamma_{A U}\left(x_{i}\right)=\sup \left(\gamma_{A}\left(x_{i}\right)\right) .
\end{gathered}
$$

Then $A^{c}=\left\{\left(x_{i},\left[\gamma_{A L}\left(x_{i}\right), \gamma_{A U}\left(x_{i}\right)\right],\left[\mu_{A L}\left(x_{i}\right), \mu_{A U}\left(x_{i}\right)\right]\right) \mid x \in X\right\}$ and by using the axiom 1 and axiom 2 , we conclude that $H_{\alpha}^{\beta}=\bar{H}_{\alpha}^{\beta}$.

Where, $\bar{H}_{\alpha}^{\beta}$ is the complement of $H_{\alpha}^{\beta}$.

(4) Let us consider the two IVIFS $A$ and $B$ and if $A \subset B$, then $\mu_{A L}\left(x_{i}\right) \leq \mu_{B L}\left(x_{i}\right), \mu_{A U}\left(x_{i}\right) \leq \mu_{B U}\left(x_{i}\right)$ and $\gamma_{A L}\left(x_{i}\right) \leq \gamma_{B L}\left(x_{i}\right), \gamma_{A U}\left(x_{i}\right) \leq \gamma_{B U}\left(x_{i}\right)$. So,

$\left((1-\alpha)\left(\mu_{B L}\left(x_{i}\right)-\mu_{A L}\left(x_{i}\right)\right)+\alpha\left(\mu_{B U}\left(x_{i}\right)-\mu_{A U}\left(x_{i}\right)\right)\right.$ $\left.+(1-\alpha)\left(\gamma_{B L}\left(x_{i}\right)-\gamma_{A L}\left(x_{i}\right)\right)+\alpha\left(\gamma_{B U}\left(x_{i}\right)-\gamma_{A U}\left(x_{i}\right)\right)\right) \geq 0$, for any $\alpha \in(0,1)$ and $\beta \in(0,1)$, then it can be written as $\left(\mu_{A L}\left(x_{i}\right)+\alpha \mu_{A U}\left(x_{i}\right)-\alpha \mu_{A L}\left(x_{i}\right)+\gamma_{A L}\left(x_{i}\right)+\alpha \gamma_{A U}\left(x_{i}\right)-\alpha \gamma_{A L}\left(x_{i}\right)\right)^{\beta}$ $\leq\left(\mu_{B L}\left(x_{i}\right)+\alpha \mu_{B U}\left(x_{i}\right)-\alpha \mu_{B L}\left(x_{i}\right)+\gamma_{B L}\left(x_{i}\right)+\alpha \gamma_{B U}\left(x_{i}\right)-\alpha \gamma_{B L}\left(x_{i}\right)\right)^{\beta}$.

This can be written as

$$
\left(\tilde{\mu}_{A}\left(x_{i}\right)+\tilde{\gamma}_{A}\left(x_{i}\right)\right)^{\beta} \leq\left(\tilde{\mu}_{B}\left(x_{i}\right)+\tilde{\gamma}_{B}\left(x_{i}\right)\right)^{\beta} .
$$

Let $\phi(z)=z^{\beta} e^{1-z^{\beta}}$ in order to show that the above function is increasing we have to show that $\frac{\partial \phi}{\partial z} \geq 0$ for all values of $z \in[0,1]$ 


$$
\begin{gathered}
\frac{\partial \phi}{\partial z}=\frac{\partial}{\partial z}\left(z^{\beta} e^{1-z^{\beta}}\right)=z^{\beta} \frac{\partial}{\partial z}\left(e^{1-z^{\beta}}\right)+e^{1-z^{\beta}} \frac{\partial}{\partial z}\left(z^{\beta}\right) \\
=z^{\beta} e^{1-z^{\beta}} \frac{\partial}{\partial z}\left(1-z^{\beta}\right)+e^{1-z^{\beta}} \beta z^{\beta-1} \\
=z^{\beta} e^{1-z^{\beta}}\left(-\beta z^{\beta-1}\right)+e^{1-z^{\beta}} \beta z^{\beta-1} \\
=e^{1-z^{\beta}} \beta z^{\beta-1}(1-\beta)
\end{gathered}
$$

and this function is greater than zero for all values of $Z \in$ $[0,1]$ for any $\alpha \in[0,1]$ and $\beta \in[0,1]$

from this the above function $\phi$ is an increasing function on $[0,1]$

Since

$$
0 \leq\left(\tilde{\mu}_{A}\left(x_{i}\right)+\tilde{\gamma}_{A}\left(x_{i}\right)\right)^{\beta} \leq\left(\tilde{\mu}_{B}\left(x_{i}\right)+\tilde{\gamma}_{B}\left(x_{i}\right)\right)^{\beta} \leq 1
$$

this implies

$$
\phi\left(\tilde{\mu}_{A}\left(x_{i}\right)+\tilde{\gamma}_{A}\left(x_{i}\right)\right)^{\beta} \leq \phi\left(\tilde{\mu}_{B}\left(x_{i}\right)+\tilde{\gamma}_{B}\left(x_{i}\right)\right)^{\beta}
$$

and

$$
\begin{aligned}
\left(\tilde{\mu}_{A}\left(x_{i}\right)\right. & \left.+\tilde{\gamma}_{A}\left(x_{i}\right)\right)^{\beta} e^{1-\left(\tilde{\mu}_{A}\left(x_{i}\right)+\tilde{\gamma}_{A}\left(x_{i}\right)\right)^{\beta}} \\
\leq & \left(\tilde{\mu}_{B}\left(x_{i}\right)+\tilde{\gamma}_{B}\left(x_{i}\right)\right)^{\beta} e^{1-\left(\tilde{\mu}_{B}\left(x_{i}\right)+\tilde{\gamma}_{B}\left(x_{i}\right)\right)^{\beta}}
\end{aligned}
$$

By multiplying -1 both sides we get,

$$
\begin{aligned}
-\left(\tilde{\mu}_{A}\right. & \left.\left(x_{i}\right)+\tilde{\gamma}_{A}\left(x_{i}\right)\right)^{\beta} e^{1-\left(\tilde{\mu}_{A}\left(x_{i}\right)+\tilde{\gamma}_{A}\left(x_{i}\right)\right)^{\beta}} \\
& \leq-\left(\tilde{\mu}_{B}\left(x_{i}\right)+\tilde{\gamma}_{B}\left(x_{i}\right)\right)^{\beta} e^{1-\left(\tilde{\mu}_{B}\left(x_{i}\right)+\tilde{\gamma}_{B}\left(x_{i}\right)\right)^{\beta}}
\end{aligned}
$$

By adding 1 on both sides we get,

$$
\begin{aligned}
& 1-\left(\tilde{\mu}_{A}\left(x_{i}\right)+\tilde{\gamma}_{A}\left(x_{i}\right)\right)^{\beta} e^{1-\left(\tilde{\mu}_{A}\left(x_{i}\right)+\tilde{\gamma}_{A}\left(x_{i}\right)\right)^{\beta}} \\
& \quad \leq 1-\left(\tilde{\mu}_{B}\left(x_{i}\right)+\tilde{\gamma}_{B}\left(x_{i}\right)\right)^{\beta} e^{1-\left(\tilde{\mu}_{B}\left(x_{i}\right)+\tilde{\gamma}_{B}\left(x_{i}\right)\right)^{\beta}}
\end{aligned}
$$

So, therefore $H_{\alpha}^{\beta}(A)=H_{\alpha}^{\beta}(B)$ so from above axioms of entropy the exponential entropy of IVIFS based on two parameters $\alpha$ and type $\beta$ is given by

$$
H_{\alpha}^{\beta}(A)=\frac{1}{n} \sum_{i=1}^{n}\left[1-\left(\tilde{\mu}_{A}\left(x_{i}\right)+\tilde{\gamma}_{A}\left(x_{i}\right)\right)^{\beta} e^{1-\left(\tilde{\mu}_{A}\left(x_{i}\right)+\tilde{\gamma}_{A}\left(x_{i}\right)\right)^{\beta}}\right]
$$

Therefore the proof is completed.

\section{MONOTONICITY OF EXPONENTIAL INTERVAL VALUED INTUITIONISTIC FUZZY ENTROPY OF ORDER $\alpha$ AND TYPE $\beta$.}

Here the Monotonicity of the function is proved by taking two examples. Where, each member of a monotone increasing sequence is greater than or equal to the preceding member and each member of a monotone decreasing sequence is less than or equal to the preceding member. Taking $A=\{[0.2,0.5],[0.1,0.2]\}$ and calculating entropy $\left(H_{\alpha}^{\beta}(A)\right)$ of $A$ for different values of $\alpha$ and $\beta$. So, here it is clear from the above two examples that the function is decreasing function as it has shown Monotonicity.
Table 1. For $\mathrm{A}=[0.2,0.5]$ Entropy $\left(H_{\alpha}^{\beta} A\right)$

\begin{tabular}{|c|c|c|c|c|c|}
\hline & $\beta=0.1$ & $\beta=0.2$ & $\beta=0.5$ & $\beta=0.7$ & $\beta=1$ \\
\hline$\alpha=0.1$ & 0.0056 & 0.02146 & 0.1074 & 0.2016 & 0.3422 \\
\hline$\alpha=0.2$ & 0.0045 & 0.0170 & 0.0954 & 0.1691 & 0.2936 \\
\hline$\alpha=0.3$ & 0.0036 & 0.0141 & 0.0786 & 0.1411 & 0.2499 \\
\hline$\alpha=0.4$ & 0.0029 & 0.0114 & 0.0643 & 0.1168 & 0.2106 \\
\hline$\alpha=0.5$ & 0.0023 & 0.0091 & 0.0523 & 0.0959 & 0.1756 \\
\hline$\alpha=0.6$ & 0.0019 & 0.0073 & 0.0420 & 0.0778 & 0.1446 \\
\hline$\alpha=0.7$ & 0.0015 & 0.0057 & 0.0334 & 0.0623 & 0.1173 \\
\hline$\alpha=0.8$ & 0.0011 & 0.0044 & 0.0261 & 0.0490 & 0.0934 \\
\hline$\alpha=0.9$ & 0.0009 & 0.0034 & 0.0199 & 0.0377 & 0.0727 \\
\hline$\alpha=1.0$ & 0.0006 & 0.0025 & 0.0149 & 0.0283 & 0.0551 \\
\hline
\end{tabular}

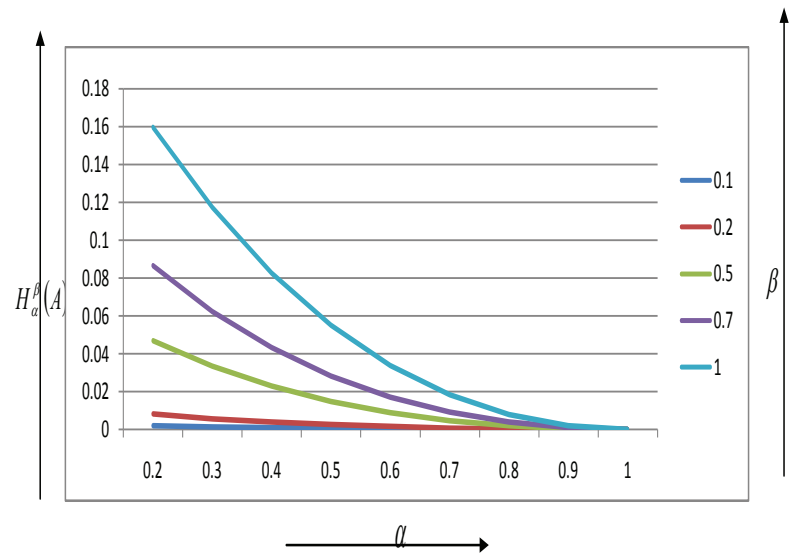

Fig. 1. Graph for $A=[0.2,0.5]$ Entropy $\left(H_{\alpha}^{\beta} A\right)$

Table 2. For $A=[0.1,0.2]$ Entropy $\left(H_{\alpha}^{\beta} A\right)$

\begin{tabular}{|c|c|c|c|c|c|}
\hline & $\beta=0.1$ & $\beta=0.2$ & $\beta=0.5$ & $\beta=0.7$ & $\beta=1$ \\
\hline$\alpha=0.1$ & 0.0029 & 0.0114 & 0.0643 & 0.1168 & 0.2106 \\
\hline$\alpha=0.2$ & 0.0021 & 0.0082 & 0.0469 & 0.0865 & 0.1596 \\
\hline$\alpha=0.3$ & 0.0015 & 0.0057 & 0.0334 & 0.0623 & 0.1173 \\
\hline$\alpha=0.4$ & 0.0010 & 0.0039 & 0.0229 & 0.0431 & 0.0827 \\
\hline$\alpha=0.5$ & 0.0006 & 0.0025 & 0.0149 & 0.0283 & 0.0551 \\
\hline$\alpha=0.6$ & 0.0004 & 0.0015 & 0.0089 & 0.0172 & 0.0339 \\
\hline$\alpha=0.7$ & 0.0002 & 0.0008 & 0.0047 & 0.0092 & 0.0183 \\
\hline$\alpha=0.8$ & 0.0001 & 0.0003 & 0.0020 & 0.0039 & 0.0078 \\
\hline$\alpha=0.9$ & 0.0000 & 0.0001 & 0.0005 & 0.0009 & 0.0019 \\
\hline$\alpha=1.0$ & 0.0000 & 0.0000 & 0.0000 & 0.0000 & 0.0000 \\
\hline
\end{tabular}

\section{MULTIPLE ATTRIBUTE DECISION MAKING METHOD}

Here, a multiple attribute decision making method using entropybased attribute weights with alternatives on attributes denoted by IVIFS is taken, and the attribute weights information for alternatives is unknown. Let $A=\left[A_{1}, A_{2}, A_{3}, \ldots, A_{m}\right]$ be a discrete set of alternatives, and $G=\left[G_{1}, G_{2}, G_{3}, \ldots, G_{n}\right]$ be the set of at- 


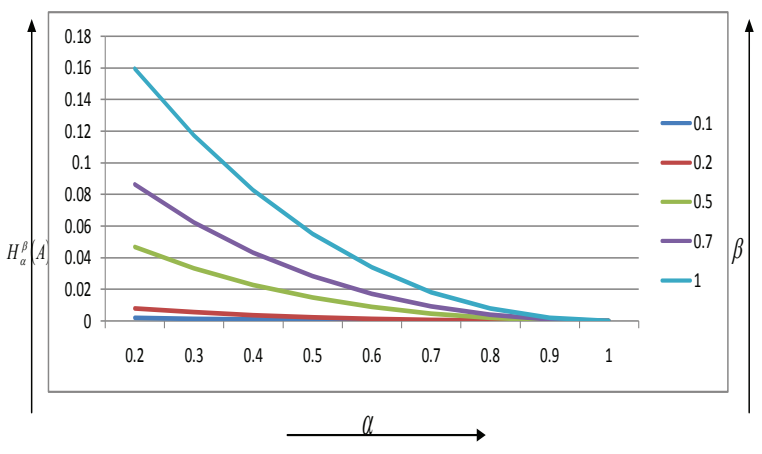

Fig. 2. Graph for $\mathrm{A}=[0.1,0.2]$ Entropy $\left(H_{\alpha}^{\beta} A\right)$

tributes. The IVIFS decision D of A on G can be written as under:-

$$
D=\left[\begin{array}{cccc}
a_{11} & a_{12} & \cdots & a_{1 n} \\
a_{21} & a_{22} & \cdots & a_{2 n} \\
\vdots & \vdots & \vdots & \vdots \\
a_{m 1} & a_{m 2} & \cdots & a_{m n}
\end{array}\right]
$$

where

$a_{i j}=\left[\left(\mu_{i j}^{-}, \mu_{i j}^{+}\right)\left(\gamma_{i j}^{-}, \gamma_{i j}^{+}\right)\right],[i=1,2,3, \cdots m ; j=1,2,3, \cdots n]$ defines an IVIFS value. We propose a method of MADM based on the proposed entropy formula.

Step 1. Normalize each attribute value $\tilde{a}_{i j}$ in the matrix $D$ into a corresponding element in the matrix $R=\left(\tilde{r}_{i j}\right)_{m \times n}=$ $\left[\left(\mu_{i j}^{-}, \mu_{i j}^{+}\right)\left(\gamma_{i j}^{-}, \gamma_{i j}^{+}\right)\right]_{m \times n}$. Considering there are two types of attributes i.e. benefit attributes and cost attributes. The normalized method is shown as under according to [19]:-

$\tilde{\mu}_{-\overline{i j}}=\frac{\mu_{-}}{\sqrt{\sum_{i=1}^{n}\left(2-\gamma_{--}-\gamma_{+j}\right)^{2}}} \tilde{\mu}_{i j}=\frac{\mu_{+}}{\left.\sqrt{\sum_{i j}^{n}\left(2-\gamma_{-1}-\gamma_{+j}\right.}\right)^{2}}$

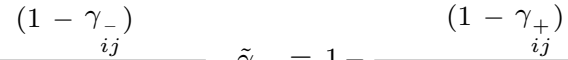

$$
\begin{aligned}
& \tilde{\gamma}_{i j}=1-\frac{i j}{\sqrt{\sum_{i=1}^{n}\left(\mu_{-\bar{j}}+\mu_{+}\right)^{2}}} \tilde{\gamma}_{i j}=1-\frac{i j}{\sqrt{\sum_{i=1}^{n}\left(\begin{array}{c}
\mu_{-}+\mu_{+} \\
i j
\end{array}\right)^{2}}}
\end{aligned}
$$

For benefit attributes $G_{j}, i=1,2,3, \cdots m ; j=$ $1,2,3, \cdots n$;

$$
\tilde{\mu}_{i j}=\frac{\left(1-\gamma_{i j}^{-}\right)^{-1}}{\sqrt{\sum_{i=1}^{n}\left(\left(\frac{1}{\mu_{-}}\right)+\left(\frac{1}{\mu_{+}}\right)\right)^{2}}} \tilde{\mu}_{i j} \tilde{i j}^{+}=\frac{\left(1-\gamma_{i j}^{+}\right)^{-1}}{\sqrt{\sum_{i=1}^{n}\left(\left(\frac{1}{\mu_{-}}\right)+\left(\frac{1}{\mu_{+}}\right)\right)^{2}}}
$$

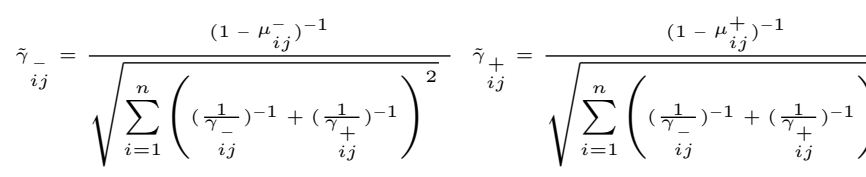

For cost attributes $G_{j}, i=1,2,3, \cdots, m ; j=1,2,3, \cdots, n$;.
Table 3. Linguistic terms for rating the alternatives

\begin{tabular}{|c|c|}
\hline Linguistic Terms & IVIFNn \\
\hline Extremely good/Extremely High/Extremely Strong & {$[1,1],[0,0]$} \\
\hline Very Very good/Very Very high/Very Very strong & {$[0.8,0.9],[0.05,0.1]$} \\
\hline Very good/Very high/Very Strong & {$[0.7,0.8],[0.1,0.2]$} \\
\hline Good/High/Strong & {$[0.6,0.7],[0.2,0.3]$} \\
\hline Medium & {$[0.4,0.6],[0.3,0.4]$} \\
\hline Bad/Low/Weak & {$[0.3,0.4],[0.4,0.5]$} \\
\hline Very Very Bad/Very Very Low/Very Very Weak & {$[0.2,0.3],[0.5,0.6]$} \\
\hline Extremely Bad/Extremely Low/Extremely Week & {$[0.1,0.2],[0.7,0.8]$} \\
\hline
\end{tabular}

Step 2. Set $\alpha \in[0,1]$ and $\beta \in[0,1]$, now on the basis of the proposed entropy in equation no. 5 , obtain the entropy matrix $E=\left(e_{i j}\right)_{m \times n}$ of the normalized decision matrix $R$, where $e_{i j}=E\left(\tilde{r}_{i j}, \alpha, \beta\right)$ for $i=1,2,3, \cdots, m ; j=1,2,3, \cdots, n$; then the information entropy of attribute $G_{j}$ defined as under [19]:-

$$
E_{j}=\frac{1}{m} \sum_{i=1}^{m} e_{i j}
$$

Then the attribute weight $W_{j},(j=1,2,3, \cdots, n)$ can be calculated as under

$$
W_{j}=\frac{1-E_{j}}{\sum_{j=1}^{n} 1-E_{j}}
$$

To provide useful information to decision maker, the entropy value across alternatives should be smaller. Therefore, the attribute should be assigned a bigger weight otherwise, such an attribute will be judged unimportant by decision maker i.e. such attributes be assigned a very small weight [20]

Step 3. On the basis of attribute weights obtained in step no. 2, obtain the weighted arithmetic average value expressed by $\gamma_{i}=$ $\left[\left(a_{i}, b_{i}\right),\left(c_{i}, d_{i}\right)\right]$ for $a_{i},(i=1,2,3, \cdots, m)$ using the interval valued Intuitionistic fuzzy weighted averaging (IIFWA) Operator [16]

$$
\begin{aligned}
\omega_{i} & =\operatorname{IIFWA} w\left(\tilde{r}_{i 1}, \tilde{r}_{i 2}, \tilde{r}_{i 3}, \cdots, \tilde{r}_{i n}\right) \\
& =\omega_{1} \tilde{r}_{i 1} \oplus \omega_{2} \tilde{r}_{i 2} \oplus \cdots \omega_{n} \tilde{r}_{i n} \\
& =\left[( 1 - \coprod _ { j = 1 } ^ { n } ( 1 - \tilde { \mu } _ { i j } ^ { - } ) ^ { \omega j } , 1 - \coprod _ { j = 1 } ^ { n } ( 1 - \tilde { \mu } _ { i j } ^ { + } ) ^ { \omega j } ) \ldots \left(\coprod_{j=1}^{n}\left(\tilde{\gamma}_{i j}^{-}\right)^{\omega j}, \coprod_{j=1}^{n}\left(\tilde{\gamma}_{i j}^{+}\right)\right.\right.
\end{aligned}
$$

Step 4. Now, calculate the scores $S\left(\tilde{\omega}_{i}\right)(i=1,2,3, \cdots, m)$ of overall collective Intuitionistic fuzzy preference values $\tilde{\omega}(i=1,2,3, \ldots, m)$ where $S\left(\tilde{\omega}_{i}\right)$ is defined as $S\left(\tilde{\omega}_{i}\right)=$ $\frac{1}{2}\left(a_{i}-b_{i}+c_{i}-d_{i}\right)$

Step 5. Now, rank all the alternatives $\omega_{i}(i=1,2,3, \cdots, m)$ and then select the best one(s) in accordance with $S\left(\tilde{\omega}_{i}\right)$.

\section{NUMERICAL EXAMPLE}

Here an example to study the effect of pollution on living being present on earth is taken. Generally, the accurate attribute value ${ }_{2}^{2}$ pollutants is very difficult to measure but the people dealing in interval valued Intuitionistic fuzzy language can easily understand the terms like "very good", "good", "medium", "bad", "very bad", rather than the accurate real numbers as given in table no.3. 
Table 4. Linguistic terms for rating human beings

\begin{tabular}{|c|c|c|c|}
\hline Affected & $\left(C_{1}\right)$ & $\left(C_{2}\right)$ & $\left(C_{3}\right)$ \\
\hline$\left(L_{1}\right)$ & $V V L$ & $V G$ & $G$ \\
\hline$\left(L_{2}\right)$ & $V L$ & $V B$ & $V H$ \\
\hline$\left(L_{3}\right)$ & $M$ & $V L$ & $S$ \\
\hline
\end{tabular}

The living beings are divided into three broader categories that are getting affected Humans $\left(L_{1}\right)$ Animals and Birds $\left(L_{2}\right)$, Plants and Forests $\left(L_{3}\right)$ and these are affected by the various attributes of pollution i.e. water pollution $\left(C_{1}\right)$ Deforestation $\left(C_{2}\right)$ and Industrial pollution $\left(C_{3}\right)$ which can be represented by interval Intuitionistic fuzzy language given in the following table no. 4.

For the example taken the decision matrix $D=\left(a_{i j}\right)_{3 \times 3}$ is listed below.

$D=\left[\begin{array}{ccc}{[0.1,0.2][0.7,0.8]} & {[0.7,0.8][0.1,0.2]} & {[0.4,0.6][0.3,0.4]} \\ {[0.2,0.3][0.5,0.6]} & {[0.2,0.3][0.5,0.6]} & {[0.8,0.9][0.05,0.1]} \\ {[0.4,0.6][0.3,0.4]} & {[0.3,0.4][0.4,0.5]} & {[0.7,0.8][0.1,0.2]}\end{array}\right]$

Step no. 1. Firstly calculate the normalized decision matrix R:

$R=\left[\begin{array}{lll}{[0.06,0.12][0.74,0.83]} & {[0.32,0.36][0.48,0.54]} & {[0.14,0.21][0.72,0.76]} \\ {[0.12,0.18][0.57,0.66]} & {[0.09,0.14][0.71,0.77]} & {[0.28,0.32][0.62,0.64]}\end{array}\right]$ $R=\left[\begin{array}{lll}{[0.12,0.18][0.57,0.66]} & {[0.09,0.14][0.71,0.77]} & {[0.28,0.32][0.62,0.64]} \\ {[0.24,0.36][0.40,0.48]} & {[0.14,0.18][0.65,0.71]} & {[0.25,0.28][0.64,0.68]}\end{array}\right]$

Step no. 2. Taking $\alpha=0.5, \beta=0.5$, then calculate the entropy matrix $\tilde{E}_{\alpha}^{\beta}$ of the normalized decision matrix R:

$$
E_{\alpha}^{\beta}=\left[\begin{array}{lll}
0.0021 & 0.0003 & 0.0009 \\
0.0088 & 0.0031 & 0.0007 \\
0.0107 & 0.0037 & 0.0008
\end{array}\right]
$$

Step no. 3. Then calculate the entropy vector of attribute $G_{j}(j=1,2,3)$ :

$$
\tilde{E} \tilde{V}_{\alpha}^{\beta}=\left[\begin{array}{lll}
0.0072 & 0.0024 & 0.0008
\end{array}\right]
$$

Then calculate the attribute weight vector:

$$
\omega=\left[\begin{array}{lll}
0.3321 & 0.3337 & 0.3342
\end{array}\right]
$$

Step no. 4. Now obtain the weighted arithmetic average value using interval-valued Intuitionistic fuzzy weighted averaging (IIFWA) operator expressed as $\omega_{i}=\left(\left[a_{i}, b_{i}\right],\left[c_{i}, d_{i}\right]\right)$ for $A_{i}(i=1,2,3)$, which are as under:

$$
\begin{aligned}
& \omega_{1}=[0.1792,0.2371][0.3649,0.3033] \\
& \omega_{2}=[0.1692,0.2157][0.3713,0.3160] \\
& \omega_{3}=[0.2103,0.2679][0.4588,0.3845]
\end{aligned}
$$

Step no. 5. Now calculate the scores $S\left(\omega_{i}\right)(i=1,2,3)$ of the collective preference value $\omega_{i}(i=1,2,3)$, which are as under:

$$
S\left(\omega_{1}\right)=-0.1260, \quad S\left(\omega_{2}\right)=-0.1512, \quad S\left(\omega_{3}\right)=-0.1826
$$

Step no. 6. Now rank all the alternatives $A_{i}(i=1,2,3)$ in accordance with the score $S\left(\omega_{i}\right)(i=1,2,3)$ of the collective preference value $\omega_{i}(i=1,2,3)$ and get the ranking order for $\alpha=$ $0.5, \beta=0.5$, as $S\left(\omega_{1}\right) \succ S\left(\omega_{2}\right) \succ S\left(\omega_{3}\right)$ and the best alternative is $S\left(\omega_{1}\right)$.

If Li's method [21] is applied to the example taken to find out affect of pollutant on various entities, the ranking order of all the alternatives is $S\left(\omega_{1}\right) \succ S\left(\omega_{2}\right) \succ S\left(\omega_{3}\right)$ and the most desirable alternative is $S\left(\omega_{1}\right)$ and also by applying Xu's method [22] to the example, the ranking order of all the alternatives is $S\left(\omega_{1}\right) \succ S\left(\omega_{2}\right) \succ S\left(\omega_{3}\right)$ and the most desirable alternative is
$S\left(\omega_{1}\right)$. In all the methods as the most desirable alternative comes out to be $S\left(\omega_{1}\right)$. Li's method [21] is only effective in solving the decision making problem with both alternatives on attributes and attribute weights information denoted by IVIFSs. Another method proposed by Boran et al. [23] utilized the definition of IVIFS to calculate the attribute weights in decision making problems under IVIF environment, where the IVIF decision matrix is not considered for decision making. The entropy based attribute weights method proposed in this paper not only is an objective calculation method but also takes into account all the alternatives on attributes.

\section{CONCLUSIONS AND FUTURE WORK}

In this paper, a new entropy on Interval-Valued Intuitionistic Fuzzy Sets (IVIFSs) is proposed along with their proofs of validity, which depends on two parameters order $\alpha$ and type $\beta$ which covers multiplicative and additive factors on interval - valued intuitionistic fuzzy sets (IVIFSs) as well as function is decreasing function as it has shown monotonicity. Then, to take a decision with unknown attribute weight, a multi attribute decision making method based on similarity measure using entropy based attribute. Finally, an example of environment has been given to show the efficiency of the algorithm developed. Future research will be to obtain better results in many fields like medical diagnosis, image processing and decision making taking into account the proposed entropy.

\section{REFERENCES}

[1] K. Atanassov, Intuitionistic fuzzy set, Fuzzy Sets and Systems, 1986, vol.30, no.1, 87-96.

[2] K. Atanassov and G. Gargov, " Interval valued Intuitionistic fuzzy set," Fuzzy Sets and Systems,1989, vol.31, no.3, pp. 343-349.

[3] L. A. Zadeh, Fuzzy Sets, Information and Control, 8, 338 353.

[4] Bustince, H., Burillo, P., Entropy on Intuitionistic fuzzy sets and on interval-valued fuzzy sets, Fuzzy Sets and Systems, 1996, 78, 305-316.

[5] Ying Jun Zhang, Pei Jun Ma, Xiao Hong Su,Chi Ping Zhang "Entropy on Interval-valued Intuitionistic Fuzzy Sets and Its Application in Multi-attribute Decision Making" International Conference on Information Fusion, 2011.

[6] A. De Luca and S. Termini, A Definition of a Nonprobabilistic Entropy in the Setting of fuzzy sets theory, Information and Control, 1972, 20, $301-312$.

[7] Kaufmann, A., Introduction to the Theory of Fuzzy Subsetsvol. 1, Fundamental Theoretical Elements, Academic Press, New York, 1975

[8] Hung W., A note on entropy of intuitionistic fuzzy sets. International Journal of Uncertainty, Fuzziness and KnowledgeBased Systems, 2003, 11, 627-633.

[9] Zhang H., Zhang W., Mei C., Entropy of interval-valued fuzzy sets based on distance and its relationship with similarity measure. Knowledge- Based Systems, 2009, 22, 449-454.

[10] Vlachos I., Sergiadis G., Intuitionistic fuzzy information- Applications to pattern recognition. Pattern Recognition Letters, 2007, 28(2): 197-206

[11] Zeng, W., Yu F., Yu X., Chen H. and Wu S., Entropy of Intuitionistic fuzzy set based on similarity measure. International Journal of Innovative Computing, Information and Control, 2009, 5(12): 4737-4744. 
[12] Yager, R.R., On the measure of fuzziness and negation. Part I: Membership in the unit interval, Intermat. J. General Systems, 5(1979), 189-200.

[13] Hwang C L, Yoon K. Multiple attribute decision making methods and applications. Springer-Verlag, Berlin, 1981.

[14] Yoon K., The propagation of errors in multiple attribute decision analysis: a practical approach. Journal of the Operational Research Society, 1989, 40: 681-686.

[15] Edwards W., How to use multi-attribute utility measurement for social decision making. IEEE Trans. on Systems, Man and Cybernetics, 1977, 7: 326-340.

[16] Saaty T L., A scaling method for priorities in hierarchical structures. Journal of Mathematical Psychology, 1977, 15: 234-281.

[17] Yang J B, Xu D L., Nonlinear information aggregation via evidential reasoning in multi-attribute decision analysis under uncertainty. IEEE Trans. On Systems, Man and CyberneticsPart A, 2002, 32: 376-393.

[18] Zeng, W.Y., Li, H.X., Inclusion measures, similarity measures and the fuzziness of fuzzy sets and their relations, International Journal of Intelligence Systems,2006, 21, 639-653.

[19] Qi X.W., Liang C.Y., Zhang E.Q., Ding Y., Approach to interval valued intuitionistic fuzzy multiple attributes group decision making based on maximum entropy. Systems Engineering-Theory and Practice, 2011, 31(10):1940-1948.

[20] Ye J., Multiple Attribute Group Decision-Making Methods with Completely Unknown Weights in Intuitionistic Fuzzy Setting and Interval- Valued Intuitionistic Fuzzy Setting. Group Decis. Negot., 2011, DOI 10.1007/s 10726- 011-92555.

[21] D. Li, "TOPSIS-Based Nonlinear-Programming Methodology for Multi-attribute Decision Making With IntervalValued Intuitionistic Fuzzy Sets," IEEE Transactions on Fuzzy Systems, 2010, vol.18, no.2, pp. 299-311.

[22] Z.Xu, Intuitionistic Fuzzy Information Aggregation Theory and Application. Science Press, BeiJing, 2008.

[23] F. Boran, S. Genc, M. Kurt and D. Akay, "A multi-criteria Intuitionistic fuzzy group decision making for supplier selection with TOPSIS method," Expert Systems with Applications, 2009, vol.36, no.8, pp. 11363-11368. 